\title{
The transition from Population III to normal galaxies: Ly $\alpha$ and He II emission and the ionising properties of high redshift starburst galaxies ${ }^{\star}$
}

\author{
D. Schaerer ${ }^{\star \star}$
}

Observatoire Midi-Pyrénées, Laboratoire d'Astrophysique, UMR 5572, 14 Av. E. Belin, 31400 Toulouse, France

Received 8 August 2002 / Accepted 11 October 2002

\begin{abstract}
Using new sets of stellar evolution models at very low metallicities $\left(Z=10^{-7}, 10^{-5}\right)$ and previously published grids we examine spectral properties of the ionising continua, the Lyman-break, and the Ly $\alpha$ and He II $\lambda 1640$ recombination lines in starbursts. The metallicity dependence of these properties, especially the transition from primordial galaxies (Population III) to currently observed metallicities, is examined for various IMFs and star formation histories. For the average properties of starbursts, approximated by a model with constant star formation, the main findings are:

- The Lyman continuum flux $Q(\mathrm{H})$ increases with decreasing metallicity. For a universal Salpeter IMF from 1-100 $M_{\odot}$ the enhancement reaches typically a factor of $\sim 3$ between solar metallicity and Pop III objects.

- While for metallicities $Z \gtrsim 1 / 50 Z_{\odot}$ the amplitude of the Lyman-break depends little on $Z$, a reduction by a factor $\sim 2$ is found at lower metallicities, due to the strong increase of the average stellar temperature.

- Using theoretical models and empirical constraints we discuss the expected evolution of the hardness of $\mathrm{He}^{+}$to $\mathrm{H}_{\text {ionising }}$ photons, $Q\left(\mathrm{He}^{+}\right) / Q(\mathrm{H})$, with metallicity and possible uncertainties. Over the metallicity range $Z=0$ to $\sim 10^{-4}$ the hardness decreases from $\log \left(Q\left(\mathrm{He}^{+}\right) / Q(\mathrm{H})\right) \sim-1.4 \ldots-2.3$ by $\sim 1.5-2$ or more orders of magnitude, depending strongly on the upper mass cut-off of the IMF. From empirical constraints we derive a hardness $\log \left(Q\left(\mathrm{He}^{+}\right) / Q(\mathrm{H})\right) \sim-3.2$ to -2.6 for metal-poor starbursts $\left(1 / 25 \lesssim Z / Z_{\odot} \lesssim 1 / 4\right)$ and softer spectra for higher metallicities. We also provide a simple estimate of the possible impact of hot WR like stars on $Q\left(\mathrm{He}^{+}\right) / Q(\mathrm{H})$ at very low metallicities $\left(Z \lesssim 10^{-4}\right)$.

- Calibrations for star formation rate determinations from various recombination lines at all metallicities and for various IMFs are derived.
\end{abstract}

For young bursts the maximum $\operatorname{Ly} \alpha$ equivalent width is shown to increase strongly with decreasing metallicity from $W(\operatorname{Ly} \alpha) \sim$ 250-350 $\AA$ at $Z \gtrsim 1 / 50 Z_{\odot}$ to $400-850 \AA$ or higher at $Z$ between $10^{-5}$ and 0 (Pop III) for the same Salpeter IMF. However, for well known reasons, the Ly $\alpha$ emission predicted likely represents an upper limit. Non-negligible He II $\lambda 1640$ emission due to stellar photoionisation appears to be limited to very small metallicities $\left(\log \left(Z / Z_{\odot}\right) \lesssim-5.3\right)$ and Population III objects. The predictions, available on the Web through the CDS and at http://webast.ast.obs-mip.fr/sfr/, should be useful for a variety of studies regarding high redshift galaxies, cosmological reionisation, and others.

Key words. cosmology: early Universe - galaxies: stellar content - stars: general - stars: fundamental parameters stars: atmospheres

\section{Introduction}

The discovery of numerous high redshift galaxies provides a unique opportunity to study galaxies in formation in the early Universe. Most of these galaxies show signs of actively ongoing massive star formation, as revealed by their overall spectral appearance, by detailed spectral features, and in many cases by the presence of strong emission lines.

* The catalog is only available in electronic form at the CDS via anonymous ftp to cdsarc.u-strasbg.fr $(130.79 .128 .5)$ or via http://cdsweb.u-strasbg.fr/cgi-bin/qcat?J/A+A/397/527

$\star \star$ e-mail: schaerer@ast.obs-mip.fr
In fact, since the detection of Lyman-break galaxies at $z \sim$ 2-4 by colour selection techniques (Steidel et al. 1996; review by Stern \& Spinrad 1999), Ly $\alpha$ surveys or other search techniques have found a large number of objects at higher redshift showing in most cases intense line emission (e.g. Hu et al. 1998, 1999; Kudritzki et al. 2000; Rhoads \& Malhotra 2001; Malhotra \& Rhoads 2002; Ellis et al. 2002; Frye et al. 2002; Ajiki et al. 2002). This also includes the most distant galaxy known to date, a lensed galaxy at $z=6.56$ found through its Ly $\alpha$ emission (Hu et al. 2002).

It is possible that such strong line emitters showing also relatively little continuum light may represent the earliest stages 
of galaxy formation, where small amounts of metals have so far been formed. Strong ongoing star formation and a small dust content, which can suppress Ly $\alpha$ emission, would then explain the high observed Ly $\alpha$ equivalent widths (cf. Hu et al. 1998). More striking is the suggestion of Malhotra \& Rhoads (2002) that the high Ly $\alpha$ equivalent widths observed in the LALA survey at $z=4.5$ could, among other explanations, be due to metal-free (so-called Population III, hereafter Pop III) objects. Possibly we are beginning to probe distant chemically little evolved galaxies, closing the gap between the first (primordial) galaxies and the high (close to solar) metallicities of massive galaxies in the local Universe.

To properly study these objects appropriate spectral models are necessary. They should take into account all possible metallicities, and also probable systematic changes of the stellar initial mass function (IMF) at very low metallicity (Abel et al. 1998; Bromm et al. 1999; Nakamura \& Umemura 2001; Hernandez \& Ferrara 2001). Providing such model calculations is the main aim of the present work.

Other applications also require an understanding of how properties like line emission and the ionising fluxes of starburst behave in the transition between metal-free (Pop III) objects and metal-poor galaxies with observable counterparts in the local Universe. This is the case in studies addressing the re-ionisation history of the Universe (e.g. Gnedin 1998; Ciardi et al. 2000; review by Loeb \& Barkana 2000), especially if account is taken for the simultaneous metal-enrichment (cf. Gnedin \& Ostriker 1997; Ferrara \& Schaerer 2002). Also, for searches of primordial galaxies, it is of interest to explore how far extreme properties such as strong He II emission predicted for Pop III starbursts (Tumlinson \& Shull 2000; Tumlinson et al. 2001, 2002; Oh et al. 2001; Bromm et al. 2001b; Schaerer 2002) are truly limited to zero metallicity. Our model calculations, including in particular metallicities $Z=0,10^{-7}, 10^{-5}$, $4 \times 10^{-4}$ and higher, allow, for the first time, such investigations.

The present paper is structured as follows. Our models ingredients, including two new sets of stellar evolution tracks at very low metallicity, are described in Sect. 2. The predicted Lyman continuum fluxes and the properties of the Lyman-break at all metallicities are presented in Sects. 4 and 5 respectively. In Sect. 6 we discuss theoretical predictions and empirical constraints on the $\mathrm{He}^{+}$ionising flux and the hardness of the ionising spectra of starbursts at various metallicities. Finally, quantitative predictions for the Ly $\alpha$ and He II $\lambda 1640$ emission are given in Sect. 7. Section 8 summarises our main conclusions.

\section{Model ingredients}

The basic model ingredients are identical to those described in Schaerer \& Vacca (1998, hereafter SV98) and the Pop III models of Schaerer (2002a, henceforth S02). A brief summary is provided subsequently including the new features introduced in the present work.

\subsection{Atmosphere models}

Depending on the metallicity different sets of atmosphere models are used.

For metallicities $Z \leq 10^{-5}$ we follow S02 in using the grid of plane parallel non-LTE atmospheres computed with the TLUSTY code of Hubeny \& Lanz (1995) for $T_{\mathrm{eff}} \geq 20000 \mathrm{~K}$ and the plane parallel line blanketed LTE models of Kurucz (1991) with a very metal-poor composition $([\mathrm{Fe} / \mathrm{H}]=-5$.) otherwise. Possible uncertainties in the predicted ionising spectra of very metal-poor stars are discussed in S02. In comparison with the computations of S02, the use of an extended grid of TLUSTY models in the present paper leads to some small differences related to the highest energy range considered here.

For higher metallicities we follow SV98 and adopt for O stars the CoStar non-LTE models including stellar winds (Schaerer \& de Koter 1997), for Wolf-Rayet (WR) stars the pure He spherically expanding non-LTE models of Schmutz et al. (1992), and Kurucz (1991) models of appropriate metallicity otherwise.

As discussed in earlier publications (e.g. Mihalas 1978; Schaerer \& de Koter 1997; Schmutz et al. 1992; Tumlinson \& Shull 2000; Kudritzki 2002) the inclusion of non-LTE models is the most important ingredient to obtain accurate predictions for the ionising spectra of massive stars.

\subsection{Stellar tracks}

To explore a wide range of metallicities covering populations from zero metallicity (Pop III), over low metallicities ( $Z \sim 4 \times$ $10^{-4}$ ) such as observed in $\mathrm{H}$ II regions in the local Universe, up to solar metallicity $\left(Z=Z_{\odot}=0.02\right)$, we use the following stellar evolution tracks: 1$)$ the Pop III tracks covering masses from 1 to $500 M_{\odot}$ with no/negligible mass loss compiled in S02 (Marigo et al. 2001; Feijóo 1999; Desjacques 2000), 2) new main sequence stellar evolution tracks from 1 to $500 M_{\odot}$ computed with the Geneva stellar evolution code for $Z=10^{-7}$, 3) non-rotating stellar models from 2 to $60 M_{\odot}$ from Meynet \& Maeder (2002) complemented with new calculations for $1 M_{\odot}$ and 85-500 $M_{\odot}$ for $\left.Z=10^{-5}, 4\right)$ non-rotating Geneva stellar evolution tracks for masses $0.8-120 M_{\odot}$ (up to $150 M_{\odot}$ for $Z=$ $0.0004)$ from the compilation of Lejeune \& Schaerer (2001) for the remaining metallicities $Z=0.0004,0.001,0.004,0.008$, $0.02\left(=Z_{\odot}\right)$, and 0.04 . For massive stars we have adopted the high mass loss tracks in all cases, as these models reproduce best various observational constraints from the local Universe (cf. Maeder \& Meynet 1994).

Our calculations at $Z \leq 10^{-5}$ include only the H-burning phase. As He-burning is typically less than $10 \%$ of the main sequence lifetime, and is generally spent at cooler temperature, neglecting this phase should have little or no consequences on our predictions. A possible exception may be if stars at very low $Z$ are very rapid rotators (cf. Maeder et al. 1999) which could suffer from non-negligible rotationally enhanced mass loss and could therefore become hot WR-like stars (cf. Sect. 6.2).

To verify our new computations we have compared several $Z=10^{-5}$ tracks with the independent calculations of 


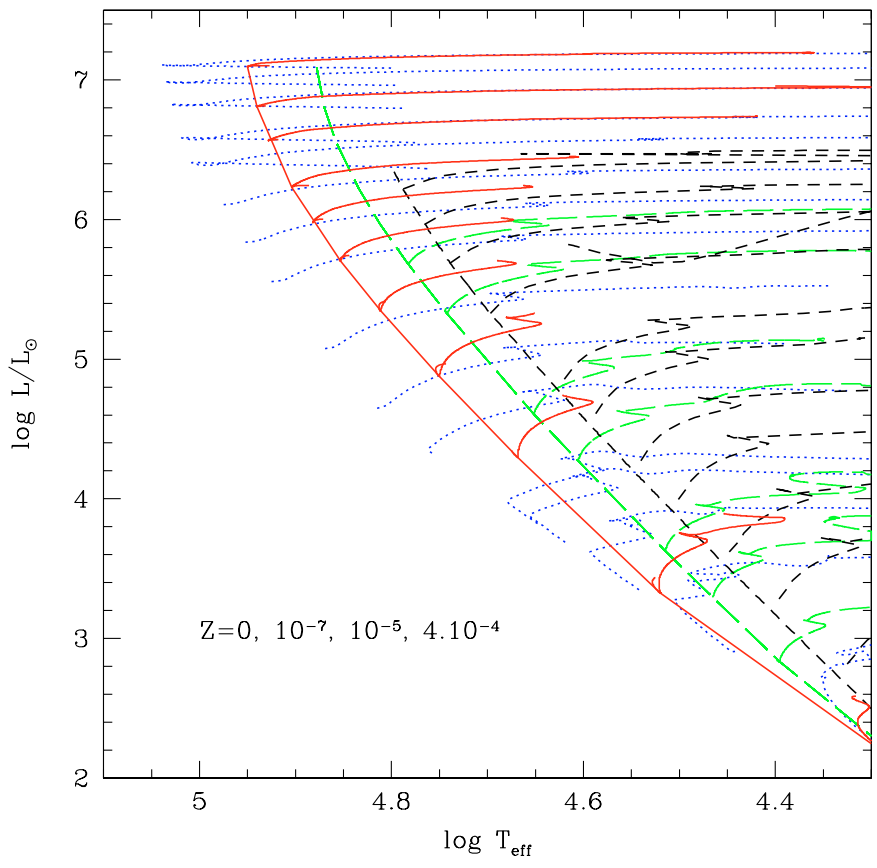

Fig. 1. HR-diagram showing the main sequence tracks of stars with masses $\gtrsim 3$ to 500 (150 for $Z=0.0004) M_{\odot}$ of metallicities $Z=0$ (Pop III, dotted line), $Z=10^{-7}$ (solid), $Z=10^{-5}$ (long-dashed), and $Z=4 \times 10^{-4}$ (short-dashed) and their ZAMS. The source of the tracks is given in Sect. 2. Note the important shift of the ZAMS to high $T_{\text {eff }}$ from low metallicity to $Z=0$.

Meynet \& Maeder (2002) done with a strongly modified version of the Geneva stellar evolution code. Good agreement is found regarding the zero age main sequences (ZAMS), Hburning lifetimes, and the overall appearance of the tracks.

The HR-diagram showing the main sequence tracks at very low metallicity is given in Fig. 1. As expected one finds an important shift of the ZAMS and main sequence toward hotter $T_{\text {eff }}$ with decreasing $Z$. For massive stars the trend shown by the low $Z$ tracks is expected to continue down to a limiting metallicity $Z_{\mathrm{lim}}$ of the order of $10^{-9}$, below which the stellar properties essentially converge to those of metal-free (Pop III) objects. This is the case, as massive stars $\left(M \gtrsim 50 M_{\odot}\right)$ with $0 \leq$ $Z<Z_{\text {lim }}$ will rapidly build up a mass fraction $X_{\mathrm{CNO}} \sim 5 \times 10^{-10}$ to $2 \times 10^{-9}$ of CNO during pre main sequence contraction or the early main sequence phase (e.g. El Eid et al. 1983; Marigo et al. 2001). A typical value of $Z_{\lim }=10^{-9}$ will be adopted subsequently for various simplified models fits.

\subsection{Evolutionary synthesis models}

As described in S02 the above stellar atmosphere models and evolutionary tracks have been included in the evolutionary synthesis code of Schaerer \& Vacca (1998). Using the prescriptions summarised below we compute the predicted properties of integrated stellar populations at different metallicities for instantaneous bursts and constant star formation, the two limiting cases of star formation histories.
Table 1. Line emission coefficients $c_{1}$ in [erg] for Case $\mathrm{B}, n_{\mathrm{e}}=$ $100 \mathrm{~cm}^{-3}$ and the different adopted $T_{\mathrm{e}}$. See S98 and S02 for sources of the atomic data.

\begin{tabular}{llll}
\hline \hline Line & $c_{1}$ & $c_{1}$ & appropriate $Q_{\mathrm{i}}$ \\
& $T_{\mathrm{e}}=30 \mathrm{kK}$ & $T_{\mathrm{e}}=10 \mathrm{kK}$ & \\
\hline Ly $\alpha$ & $1.04 \times 10^{-11}$ & $1.04 \times 10^{-11}$ & $Q(\mathrm{H})$ \\
He II $\lambda 1640$ & $5.67 \times 10^{-12}$ & $6.40 \times 10^{-12}$ & $Q\left(\mathrm{He}^{+}\right)$ \\
$\mathrm{H} \alpha$ & $1.21 \times 10^{-12}$ & $1.37 \times 10^{-12}$ & $Q(\mathrm{H})$ \\
\hline
\end{tabular}

\subsubsection{Nebular emission}

Among other predictions the code in particular computes the recombination line spectrum including Ly $\alpha$, He II $\lambda 1640$, He II $\lambda 3203, \mathrm{He}$ I $\lambda 4026, \mathrm{He}$ I $\lambda 4471$, He II $\lambda 4686, \mathrm{H} \beta$, He I $\lambda 5016, \mathrm{He}_{\mathrm{I}} \lambda 2876$, and $\mathrm{H} \alpha$. Case $\mathrm{B}$ recombination is assumed for an electron temperature of $T_{\mathrm{e}}=30000 \mathrm{~K}$ at $Z \leq 10^{-5}$ and $T_{\mathrm{e}}=10000 \mathrm{~K}$ otherwise, and a low electron density $\left(n_{\mathrm{e}}=100 \mathrm{~cm}^{-3}\right)$. Ly $\alpha$ emission is computed assuming a fraction of 0.68 of photons converted to Ly $\alpha$ (Spitzer 1978). The line emission coefficients $c_{1}$ (defined by Eq. (7)) of interest here are listed in Table 1.

Some differences, e.g. due to a more realistic temperature structure or due to collisional effects on $\mathrm{H}$ lines, can be expected between the adopted prescriptions and predictions from detailed photoionisation models (see e.g. Stasińska \& Tylenda 1986; Stasińska \& Schaerer 1999). However, for the scope of the present investigation these effects can quite safely be neglected.

As shown by $\mathrm{S} 02$ the inclusion of nebular continuous emission processes is crucial for very metal-poor objects with intense ongoing star formation. Following S02 we include freefree, free-bound, and $\mathrm{H}$ two-photon continuum emission assuming $T_{\mathrm{e}}=20000 \mathrm{~K}$ or $10000 \mathrm{~K}$ and the above value of $n_{\mathrm{e}}$.

Table 2. Summary of IMF model parameters. All models assume a Salpeter slope for the IMF.

\begin{tabular}{llrr}
\hline \hline Model ID & $M_{\text {low }}$ & $M_{\text {up }}$ & $c_{\mathrm{M}}$ \\
\hline $\mathrm{A}$ & 1 & 100 & 2.55 \\
$\mathrm{~B}$ & 1 & 500 & 2.30 \\
$\mathrm{C}$ & 50 & 500 & 14.5 \\
\hline
\end{tabular}

\subsubsection{Initial mass function}

In view of our ignorance on the massive star IMF at very low metallicities $\left(Z<4 \times 10^{-4}\right)$ we adopt as in S02 a powerlaw IMF and different upper and lower mass limits with the aim of assessing their impact on the properties of integrated stellar populations. The main cases modeled here are summarised in Table $2^{1}$. For all models the IMF slope is taken as the Salpeter value ( $\alpha=2.35$ ) between the lower and upper mass cut-off values $M_{\text {low }}$ and $M_{\text {up }}$ respectively.

\footnotetext{
1 For comparison with SFR indicators used for "normal" galaxies we also indicate the mass conversion factor $c_{\mathrm{M}}$ expressing the relative masses between our model IMF and a "standard" Salpeter IMF with $M_{\text {low }}=0.1$ and $M_{\text {up }}=100 M_{\odot}$ used frequently throughout the literature. $c_{\mathrm{M}}$ is given by $c_{\mathrm{M}}=\int_{0.1}^{100} M \Phi(M) \mathrm{d} m / \int_{M_{\mathrm{low}}}^{M_{\mathrm{up}}} M \Phi(M) \mathrm{d} m$.
} 
ZAMS, Z=0-0.02, Salpeter IMF $1-100 \mathrm{M}_{\odot}$

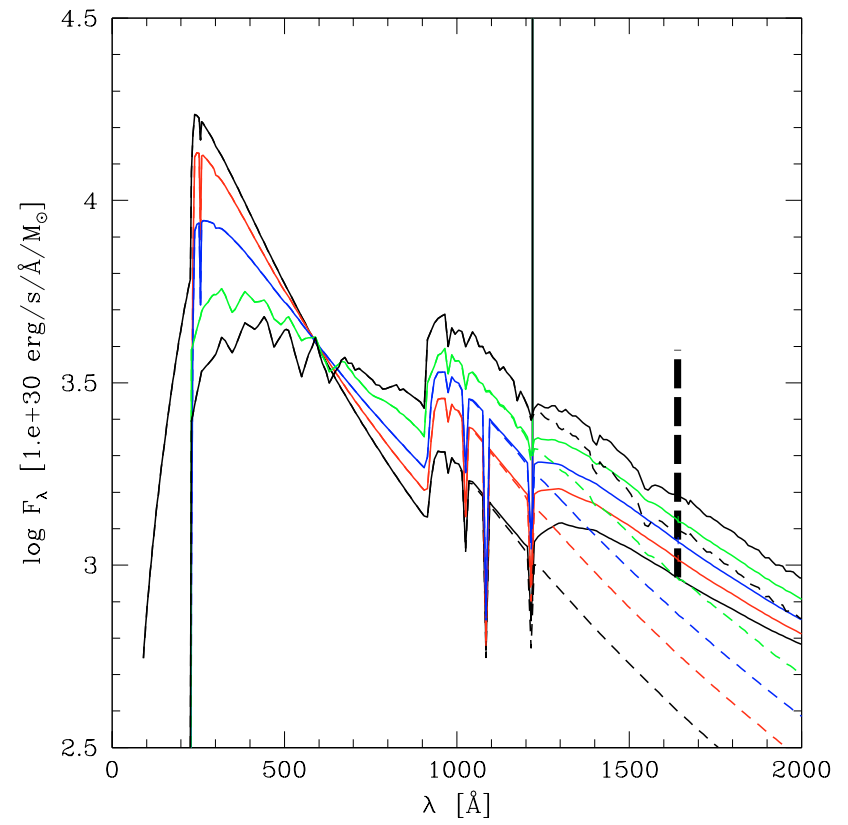

ZAMS, $Z=0-10^{-5}$, Salpeter IMF: $M_{\text {up }}=100,500 \mathrm{M}_{\odot}$

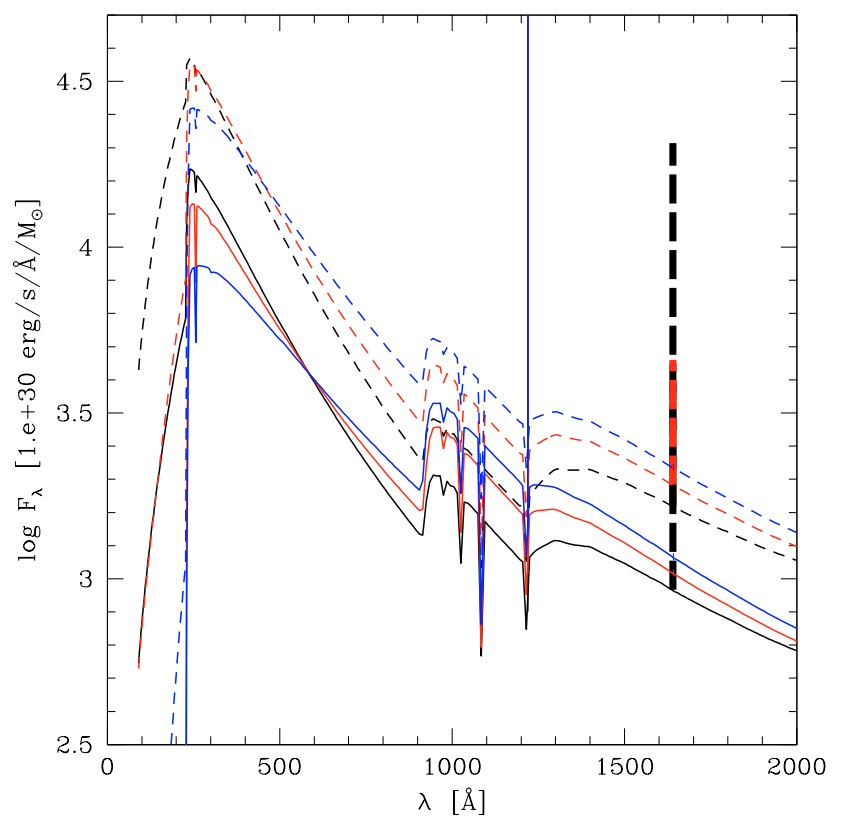

Fig. 2. Predicted SEDs including Ly $\alpha$ and He II $\lambda 1640$ emission lines for zero age main sequence (ZAMS) models at different metallicities. Left panel: dependence of the SED on metallicity: the metallicities $Z=0$. (Pop III), $10^{-7}, 10^{-5}, 0.0004$, and 0.02 (solar) are from top to bottom in the EUV $(\lambda<912 \AA)$, and reversed at longer wavelengths. The dashed lines are the pure stellar emission, the solid lines show the total (stellar + nebular) emission. IMF A in all cases. Right panel: dependence of the SED on $M_{\text {up }}$ for metallicities $Z=0$. (Pop III), $10^{-7}$, and $10^{-5}$. Solid lines show the IMF A, dashed lines the IMF B. In all cases the total (stellar + nebular) emission is shown. Discussion in text.

The model A IMF is a good description of the IMF in observed starbursts (e.g. Leitherer 1998; Schaerer 2002b) down to $\sim 1 / 50 Z_{\odot}\left(=4 \times 10^{-4}\right)$, the metallicity of I $\mathrm{Zw} 18$ representing the most metal-poor galaxy known to date. It is adopted in all calculations for $Z \geq 4 \times 10^{-4}$. IMFs B and $\mathrm{C}$, favouring the formation of very massive stars, could be representative of stellar populations at metallicities $Z \lesssim Z_{\text {crit }} \approx 10^{-5}$ (e.g. Bromm et al. 2001a), where altered fragmentation properties may form preferentially more massive stars (cf. Abel et al. 1998; Bromm et al. 1999; Nakamura \& Umemura 2001). The computations at $Z \leq 10^{-5}$ consider all IMF cases (A, B, and C). Note that our calculations obviously do not apply to cases where only one or few massive stars form within a pre-galactic halo, as suggested by the simulations of Abel et al. (2002).

\section{Overview of EUV-UV spectra of starbursts at various metallicities}

To illustrate several points discussed in detail below, we plot the EUV to UV spectral energy distribution (SED) for selected models at different metallicities and computed with different IMFs (see Fig. 2). Only ZAMS models are shown here for simplicity.

The main points apparent from this figure are:

- The rapid decrease of the $\mathrm{He}^{+}$ionising photon flux (i.e. flux above $228 \AA$ ) and the associated overall softening of the ionising spectrum from Pop III to higher metallicity (cf. Sects. 4 and 6).

- The increase of the Lyman-break amplitude with increasing $Z$ (Sect. 5).
- The rapid decrease of the contribution of nebular continuous emission to the UV continuum flux (cf. S02).

- The strong dependence of the ionising flux and in turn also of the nebular continuous emission on the upper mass cutoff of the IMF, which is considered as a free model parameter for very low ( $Z \leq 10^{-5}$, currently unobserved) metallicities (Sects. 4 and 6).

The metallicity dependence of the emission lines (strongest in the Pop III models) cannot clearly be discerned from this figure. It is discussed in Sect. 7.

\section{4. lonising properties of starbursts at various metallicities}

\subsection{Burst models}

The basic quantities describing the ionising spectrum are the emitted number of $\mathrm{H}, \mathrm{He}$, and $\mathrm{He}^{+}$ionising photons, denoted by $Q(\mathrm{H}), Q\left(\mathrm{He}^{0}\right)$ and $Q\left(\mathrm{He}^{+}\right)$respectively, and the hardness $Q\left(\mathrm{He}^{+}\right) / Q(\mathrm{H})\left(Q\left(\mathrm{He}^{0}\right) / Q(\mathrm{H})\right)$ tracing the energy range between 54 (24.6) and $13.6 \mathrm{eV}$. The predicted temporal evolution of $Q(\mathrm{H})$ is shown in Fig. 3 (upper panel) for all metallicities between $Z=0$. and $2 \times Z_{\odot}$. For the very low metallicities $\left(Z \leq 10^{-5}\right)$ only the models with an IMF extending to $500 M_{\odot}$ (model B) are shown for clarity sake. Adopting a larger value of $M_{\text {up }}$ affects only the predictions at very young ages (ages $\$ 2.5 \mathrm{Myr}$ ) due to the very short lifetime of these stars.

The predicted $Q_{\mathrm{i}}$ of ZAMS populations $($ age $=0$ ) for all IMF cases are listed in Table 3. For completeness with S02 the photon flux in the Lyman-Werner band (11.2-13.6 eV) capable to dissociate $\mathrm{H}_{2}$ is also listed. 


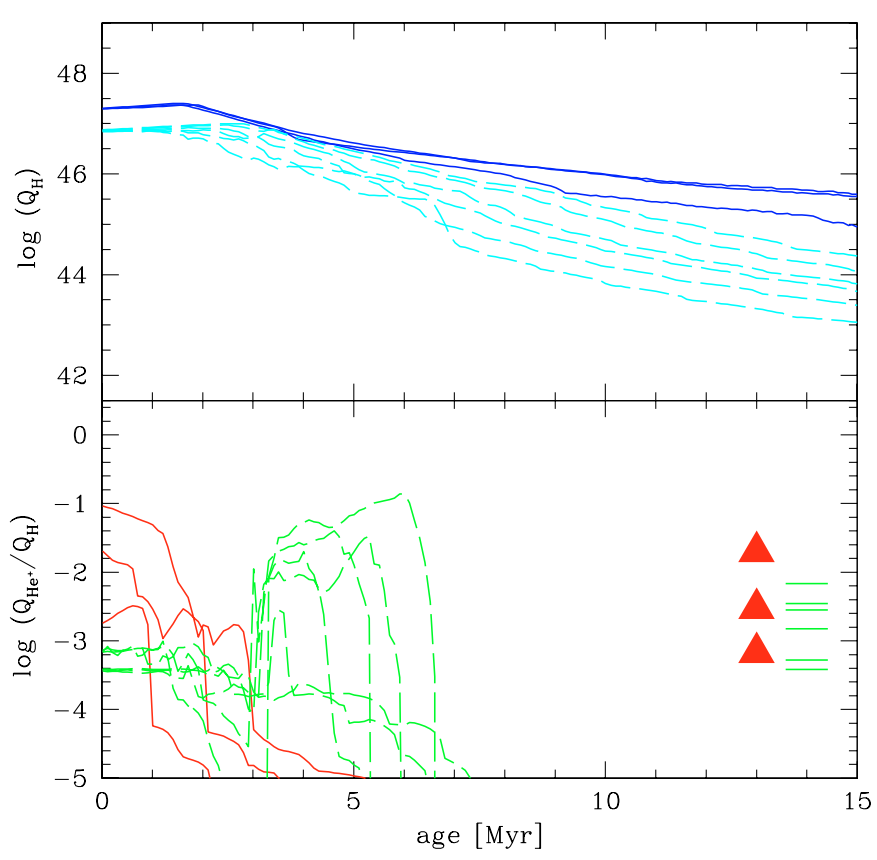

Fig. 3. Temporal evolution of the $\mathrm{H}$ ionising photon flux $Q(\mathrm{H})$ (upper panel) and the hardness $Q\left(\mathrm{He}^{+}\right) / Q(\mathrm{H})$ (lower panel) for instantaneous bursts at all metallicities $Z$ between $0.04\left(=2 Z_{\odot}\right)$ and 0 . (Pop III). The very metal-poor models $\left(Z=10^{-5}, 10^{-7}\right.$ and 0$)$ with the IMF B (1$500 M_{\odot}$ ) are shown as solid lines from top to bottom. The remaining metallicities computed for the IMF A (1-100 $\left.M_{\odot}\right)$ are shown with dashed lines. Their hardness $Q\left(\mathrm{He}^{+}\right) / Q(\mathrm{H})$ reached for constant star formation at equilibrium is shown by the solid triangles $\left(Z \leq 10^{-5}\right)$, and the short lines for larger $Z$. Predictions for IMF cases not shown here are summarised in Tables 3 and 4. Discussion in text.

The main difference in the Lyman continuum photon output at different $Z$ is a slower decline of the ionising photon production at low metallicities, due to the blueward shift of the main sequence. The temporal evolution of $Q(\mathrm{H})$ at $Z=10^{-7}$ is essentially undistinguishable from the Pop III case. The larger $Q(\mathrm{H})$ apparent for $Z \leq 10^{-5}$ at ages $\lesssim 2.5 \mathrm{Myr}$ are essentially due to the larger value of $M_{\text {up }}$ adopted at very low $Z$. The difference at older ages (when stars with masses $>100 M_{\odot}$ have disappeared) represents the pure metallicity difference.

As can be seen from Table 3 the $Q(\mathrm{H})$ production of ZAMS populations at different metallicities increases somewhat with decreasing $Z$; the changes remain fairly small $(\$ 40 \%)$ in reasonable agreement with other estimates (e.g. Tumlinson \& Shull 2000). However, in cases such as constant star formation (equivalent to a temporal average) the $Z$-dependence is more pronounced (cf. Sect. 4.2).

\subsection{Constant star formation}

The main predictions for models with constant star formation at all metallicities and for all the IMF cases are listed in Table 4. In this case most quantities of interest here reach rapidly (over timescales $\$ 6-10 \mathrm{Myr}$; except for the Lyman-break and $Q(\mathrm{LW})$ requiring $\gtrsim 200 \mathrm{Myr}$ ) an equilibrium value given in the table, normalised to a star formation rate (SFR) of $1 M_{\odot} \mathrm{yr}^{-1}$. In addition to the ionising photon production $Q_{\mathrm{i}}$ (Cols. 3-5), and the
Table 3. ZAMS properties of integrated stellar populations at various metallicities. All quantities are normalised to a total burst mass of $1 M_{\odot}$. The logarithm of the ionising photon fluxes $Q_{\mathrm{i}}$ in units of [photon s ${ }^{-1} M_{\odot}^{-1}$ ] is given.

\begin{tabular}{rrrrrr}
\hline \hline$Z$ & IMF & $Q(\mathrm{H})$ & $\begin{array}{c}Q\left(\mathrm{He}^{0}\right) \\
{\left[\log \left(\left(\mathrm{photon} \mathrm{s}^{-1}\right) /\left(M_{\odot} \mathrm{yr}^{-1}\right)\right)\right]}\end{array}$ \\
\hline 0. & A & 46.98 & 46.75 & 45.54 & 46.22 \\
0. & B & 47.29 & 47.10 & 46.26 & 46.40 \\
0. & C & 47.98 & 47.80 & 47.05 & 46.96 \\
$10^{-7}$ & A & 46.94 & 46.65 & 43.45 & 46.36 \\
$10^{-7}$ & B & 47.30 & 47.06 & 45.61 & 46.55 \\
$10^{-7}$ & C & 48.01 & 47.78 & 46.39 & 47.14 \\
$10^{-5}$ & A & 46.90 & 46.55 & 42.39 & 46.44 \\
$10^{-5}$ & B & 47.30 & 46.99 & 44.56 & 46.64 \\
$10^{-5}$ & C & 48.02 & 47.73 & 45.35 & 47.24 \\
0.0004 & A & 46.88 & 46.43 & 43.4 & 46.45 \\
0.0004 & A & 47.01 & 46.58 & 43.62 & 46.53 \\
0.001 & A & 46.86 & 46.41 & 43.44 & 46.47 \\
0.004 & A & 46.85 & 46.38 & 43.41 & 46.50 \\
0.008 & A & 46.84 & 46.35 & 43.67 & 46.52 \\
0.020 & A & 46.85 & 46.34 & 43.72 & 46.54 \\
0.040 & A & 46.87 & 46.33 & 43.71 & 46.59 \\
\hline
\end{tabular}

* Salpeter IMF with $M_{\text {up }}=150 M_{\odot}$ and $M_{\text {low }}=1 M_{\odot}$.

$\mathrm{H}_{2}$ photodissociating photon flux $(Q(\mathrm{LW}), \mathrm{Col} .6)$, we list the average energies $\bar{E}(Q(\mathrm{H}))$ and $\bar{E}\left(Q\left(\mathrm{He}^{+}\right)\right)$of the Lyman continuum photons and the photons with energies above $54 \mathrm{eV}$ (Cols. 7 and 8). These quantities, not further discussed here, are e.g. of interest to estimate the thermal evolution of the ISM. Most of the data for $Z=0,0.0004$, an 0.02 were already given in Table 3 of S02. Due to the use of a finer grid of atmosphere models at $Z=0$ some small changes are found for these models $^{2}$. The values in Table 4 supersede those of S02.

As expected from the earlier discussion (see Fig. 3), the Lyman continuum flux $Q(\mathrm{H})$ shows an increase with decreasing metallicity, which can be fitted to an accuracy better than $10 \%$ by

$\log (Q(\mathrm{H}))=-0.0029 \times(\log (Z)+9 .)^{2.5}+53.81$

valid for the Salpeter IMF from $1-100 M_{\odot}$ (models A) and $Z \in$ $\left[10^{-9}, 0.04\right]$. The limitation to $Z \geq Z_{\lim } \approx 10^{-9}$ is due to the fact that all metallicities below $Z_{\text {lim }}$ are equivalent to the Pop III $(Z=0)$ case, as discussed in Sect. 2.2. Fits for alternate IMFs may be derived from the data in Table 4.

Overall, while ZAMS Lyman continuum fluxes vary by less than $\$ 40 \%$ over the entire metallicity range for IMF A (Table 3), the ionising output at SFR = const. shows an increase of an factor $\sim 1.9$ (2.8) between solar and $1 / 50 Z_{\odot}$ (zero metallicity). Even larger increases are of course predicted in the case of IMFs extending to higher masses (models B and C).

\section{Lyman-break predictions at various metallicities}

Quantitative predictions of the amplitude of the Lyman-break, i.e. the relative flux above and below the Lyman edge are of

\footnotetext{
${ }^{2} f_{1640}$ and the corresponding $Q\left(\mathrm{He}^{+}\right)$are reduced by $\sim 9,4$, and $30 \%$ for IMFs A, B, and C respectively.
} 
Table 4. Predictions for the case of constant star formation models (equilibrium values) with different IMFs (Col. 2) at all metallicities (Col. 1). Given are the ionising photon fluxes $Q_{\mathrm{i}}$ (Cols. 3-6), their average energies $\bar{E}$ (Cols. 7 and 8), and the proportionality constants $f_{1}$ between recombination line luminosities and the SFR (cf. Eq. (7), Cols. 9-11). The logarithm of the ionising photon fluxes $Q_{\mathrm{i}}$ is given in units of [photon s ${ }^{-1} M_{\odot}^{-1}$ ].

\begin{tabular}{|c|c|c|c|c|c|c|c|c|c|c|}
\hline \multirow[t]{2}{*}{$Z$} & \multirow[t]{2}{*}{ IMF } & $Q(\mathrm{H})$ & $Q\left(\mathrm{He}^{0}\right)$ & $Q\left(\mathrm{He}^{+}\right)$ & $Q(\mathrm{LW})$ & $\bar{E}(Q(\mathrm{H}))$ & $\bar{E}\left(Q\left(\mathrm{He}^{+}\right)\right)$ & \multirow{2}{*}{\multicolumn{2}{|c|}{$\begin{array}{r}J_{\mathrm{H} \alpha} \\
{\left[\operatorname{erg~s}^{-1}\right]}\end{array}$}} & $f_{1640}$ \\
\hline & & \multicolumn{4}{|c|}{$\left[\log \left(\left(\right.\right.\right.$ photon s $\left.\left.\left.{ }^{-1}\right) /\left(M_{\odot} \mathrm{yr}^{-1}\right)\right)\right]$} & \multicolumn{2}{|c|}{$[\mathrm{eV}]$} & & & \\
\hline 0. & $\mathrm{~A}$ & 53.81 & 53.50 & 51.49 & 53.57 & 26.61 & 66.08 & $6.80 \mathrm{e}+42$ & $7.91 \mathrm{e}+41$ & $1.74 \mathrm{e}+40$ \\
\hline 0. & B & 53.93 & 53.64 & 52.23 & 53.57 & 27.78 & 68.15 & $8.86 e+42$ & $1.03 \mathrm{e}+42$ & $9.66 e+40$ \\
\hline 0. & $\mathrm{C}$ & 54.44 & 54.19 & 53.03 & 53.65 & 29.60 & 68.22 & $2.85 e+43$ & $3.32 \mathrm{e}+42$ & $6.01 e+41$ \\
\hline $10^{-7}$ & A & 53.80 & 53.43 & 50.39 & 53.67 & 25.18 & 61.68 & $6.59 \mathrm{e}+42$ & $7.67 \mathrm{e}+41$ & $1.40 \mathrm{e}+39$ \\
\hline $10^{-7}$ & B & 53.95 & 53.61 & 51.42 & 53.69 & 25.99 & 64.86 & $9.26 \mathrm{e}+42$ & $1.08 \mathrm{e}+42$ & $1.49 \mathrm{e}+40$ \\
\hline $10^{-7}$ & $\mathrm{C}$ & 54.51 & 54.21 & 52.21 & 53.84 & 27.16 & 64.80 & $3.34 \mathrm{e}+43$ & $3.89 \mathrm{e}+42$ & $9.29 \mathrm{e}+40$ \\
\hline $10^{-5}$ & A & 53.70 & 53.26 & 48.71 & 53.65 & 23.95 & 59.62 & $5.15 e+42$ & $5.99 \mathrm{e}+41$ & $2.91 e+37$ \\
\hline $10^{-5}$ & B & 53.88 & 53.48 & 50.71 & 53.69 & 24.74 & 61.77 & $7.82 \mathrm{e}+42$ & $9.10 \mathrm{e}+41$ & $2.88 \mathrm{e}+39$ \\
\hline $10^{-5}$ & $\mathrm{C}$ & 54.49 & 54.13 & 51.50 & 53.97 & 25.61 & 61.77 & $3.20 \mathrm{e}+43$ & $3.73 e+42$ & $1.82 \mathrm{e}+40$ \\
\hline 0.0004 & A & 53.63 & 53.10 & $50.08^{\dagger}$ & 53.74 & 21.62 & $52.60^{\dagger}$ & $4.38 \mathrm{e}+42$ & $5.10 \mathrm{e}+41$ & $6.79 e+38^{\dagger}$ \\
\hline 0.0004 & $\mathrm{~A}^{\star}$ & 53.70 & 53.20 & $50.42^{\dagger}$ & 53.75 & 21.96 & $61.54^{\dagger}$ & $5.22 \mathrm{e}+42$ & $6.08 \mathrm{e}+41$ & $1.50 e+39^{\dagger}$ \\
\hline 0.001 & A & 53.59 & 53.04 & $50.17^{\dagger}$ & 53.72 & 21.47 & $60.38^{\dagger}$ & $4.01 \mathrm{e}+42$ & $4.67 \mathrm{e}+41$ & $8.39 e+38^{\dagger}$ \\
\hline 0.004 & A & 53.50 & 52.93 & II & 53.67 & 21.27 & II & $3.37 \mathrm{e}+42$ & $4.36 \mathrm{e}+41$ & II \\
\hline 0.008 & A & 53.44 & 52.83 & II & 53.63 & 20.90 & II & $2.89 \mathrm{e}+42$ & $3.73 e+41$ & II \\
\hline 0.020 & A & 53.36 & 52.75 & II & 53.56 & 20.84 & II & $2.44 \mathrm{e}+42$ & $3.16 \mathrm{e}+41$ & II \\
\hline 0.040 & A & 53.28 & 52.65 & II & 53.49 & 20.88 & II & $2.00 \mathrm{e}+42$ & $2.59 \mathrm{e}+41$ & II \\
\hline
\end{tabular}

$\star$ Salpeter IMF with $M_{\text {up }}=150 M_{\odot}$ and $M_{\text {low }}=1 M_{\odot}$.

$\dagger$ uncertain predictions for reasons discussed in Sect. 6.

II no data provided, as predictions uncertain and strongly overestimated (cf. Sect. 6).

importance for example to estimate the escape fraction $f_{\text {esc }}$ of ionising photons out of their hosts. Such measurements are possible in relatively nearby $(z \gtrsim 0.015)$ and high redshift galaxies (e.g. Leitherer et al. 1995; Deharveng et al. 1997, 2001; Steidel et al. 2001).

Different working definitions of the Lyman-break exist in the literature. For simplicity we chose the definition adopted in Starburst99 (Leitherer et al. 1999), quantifying the break amplitude by $912^{+} / 912^{-}$, where $912^{+}$is the average flux in $F_{\lambda}$ units over the interval $1080-1120 \AA$, and $912^{-}$the average over 870-900 $\mathrm{A}$. A word of caution is appropriate if wavelengths $\lambda \gtrsim \lambda(\operatorname{Ly} \alpha)$ are used as reference for the red side of the Lymanbreak. In this case the continuum may, for very metal-poor objects, include a non negligible contribution from nebular continuous emission (see S02) which itself also depends on the escape fraction of Lyman continuum photons. A detailed SED fit should then be undertaken to quantify $f_{\text {esc }}$.

The temporal evolution of the Lyman-break predicted by the models at metallicities $0 \leq Z \leq Z_{\odot}$ is shown in Fig. 4 for instantaneous bursts and constant star formation. The main finding apparent here is the overall reduction of the Lyman-break at metallicities $Z \lessgtr 10^{-4 \ldots-5}$, e.g. by $0.2-0.3$ dex at very young ages, for identical IMFs or larger values of $M_{\text {up }}{ }^{3}$. This is due to the decrease of the break in individual stars with increasing $T_{\text {eff }}$ and to the shift of the main sequence towards hotter temperatures. At higher metallicity $\left(Z \gtrsim 4 \times 10^{-4}\right)$ this temperature/metallicity dependence is not important, and e.g. the predicted values at SFR $=$ const. (near equilibrium at $t \gtrsim 10^{8} \mathrm{yr}$ )

\footnotetext{
${ }^{3}$ IMFs A and B yield Lyman-break amplitudes differing by less than $\sim 0.1$ dex.
}

vary by less than $10 \%$ around $\log \left(912^{+} / 912^{-}\right) \sim 0.58$. In contrast for very low metallicities one has for the same case $\log \left(912^{+} / 912^{-}\right) \sim 0.3-0.4$ for the IMFs A and B.

Note that, as expected, at $Z \geq 10^{-3}$ our predictions at young ages are somewhat $(\sim 0.1 \mathrm{dex})$ smaller than the Starburst99 models for identical $Z$ and IMF, due to the inclusion of nonLTE O star model atmospheres in the present computations. For constant star formation this difference becomes, however, smaller. We also compared our calculations to the models of Smith et al. (2002; Norris, private communication) including more sophisticated non-LTE stellar atmospheres. For metallicities $0.001 \leq Z \leq Z_{\odot}$ and SFR = const. their Lyman-break predictions show an additional but small $(\sim 10-20 \%)$ reduction.

\section{The $Q\left(\mathrm{He}^{+}\right) / Q(\mathrm{H})$ hardness of starbursts at various metallicities}

\subsection{Predictions at metallicities $Z=0$ to $Z_{\odot}$}

\subsubsection{Burst models}

The time evolution of the predicted hardness $Q\left(\mathrm{He}^{+}\right) / Q(\mathrm{H})$ is shown in the lower panel of Fig. 3. The corresponding hardness reached at equilibrium in the case of constant star formation (cf. below), plotted on the right, illustrates the non negligible difference with the hardness derived from a simple ZAMS population neglecting stellar evolution effects (cf. S02).

Note that predicted quantities such as $Q\left(\mathrm{He}^{+}\right) / Q(\mathrm{H})$ rely obviously strongly on the adopted value of $M_{\text {up }}$. For very low $Z$ this quantitative dependence can be estimated from the tabulated ZAMS properties (Table 3 ). 


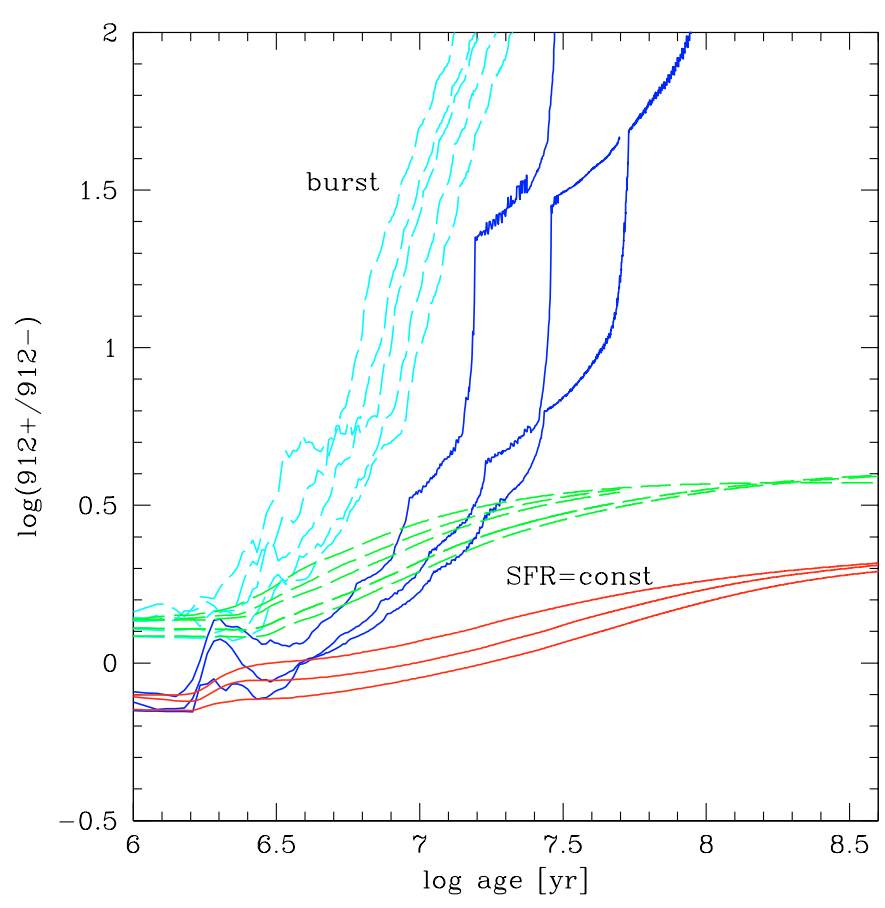

Fig. 4. Temporal evolution of the Lyman-break for instantaneous bursts and constant star formation at all metallicities $0 \leq Z \leq Z_{\odot}$. Dashed lines show the predictions for $Z \geq 4 \times 10^{-4}$, solid lines are for $Z=0$ (Pop III), $Z=10^{-7}$, and $10^{-5}$ (from bottom to top). The behaviour of the very low $Z$ burst models at ages $\log t \sim 7.2,7.5,7.7$ are artifacts due to the limited numerical resolution in tracks. Note the small sensitivity of the Lyman-break for SFR $=$ const. at metallicities $Z \geq 4 \times 10^{-4}$ and the important decrease at lower metallicities.

As expected from the strong decrease of the stellar temperatures with increasing metallicity (cf. Fig. 1) both the maximum hardness (at age $=0$ ) and the overall $Q\left(\mathrm{He}^{+}\right) / Q(\mathrm{H})$ decreases for $Z$ between 0 and $10^{-5}$. The typical timescale for a decrease of $Q\left(\mathrm{He}^{+}\right) / Q(\mathrm{H})$ by $\sim 2$ dex in a burst is driven by the redward stellar evolution, and is short ( 2-3 Myr), with obvious potential implications for the detection of sources with very hard spectra (cf. Sect. 7). Possible additional sources of $\mathrm{He}^{+}$ionising photons not included here are discussed in Sect. 6.2.

At higher metallicities $\left(Z \gtrsim 4 \times 10^{-4}\right)$ the present models predict a re-increase of $Q\left(\mathrm{He}^{+}\right) / Q(\mathrm{H})$ after $\gtrsim 3-4 \mathrm{Myr}$, due to presence of WR stars, among which a fraction is found at high temperatures (cf. Schmutz et al. 1992; SV98). Albeit with minor quantitative differences, a qualitatively similar behaviour is predicted by the Starburst 99 models based on very similar input physics (Leitherer et al. 1999). However, these predictions depend especially on the procedure adopted to link stellar tracks with atmospheres in WR phases with strong winds, and on the neglect of line blanketing in the adopted WR model atmospheres. The reality and the extent of such a trend remains therefore questionable, especially at the largest metallicities (cf. review of Schaerer 2000). Indeed using recent line blanketed $\mathrm{O}$ and WR atmospheres and different prescriptions to connect the interior and atmosphere models Smith et al. (2002) find a considerably softer spectrum - i.e. reduced

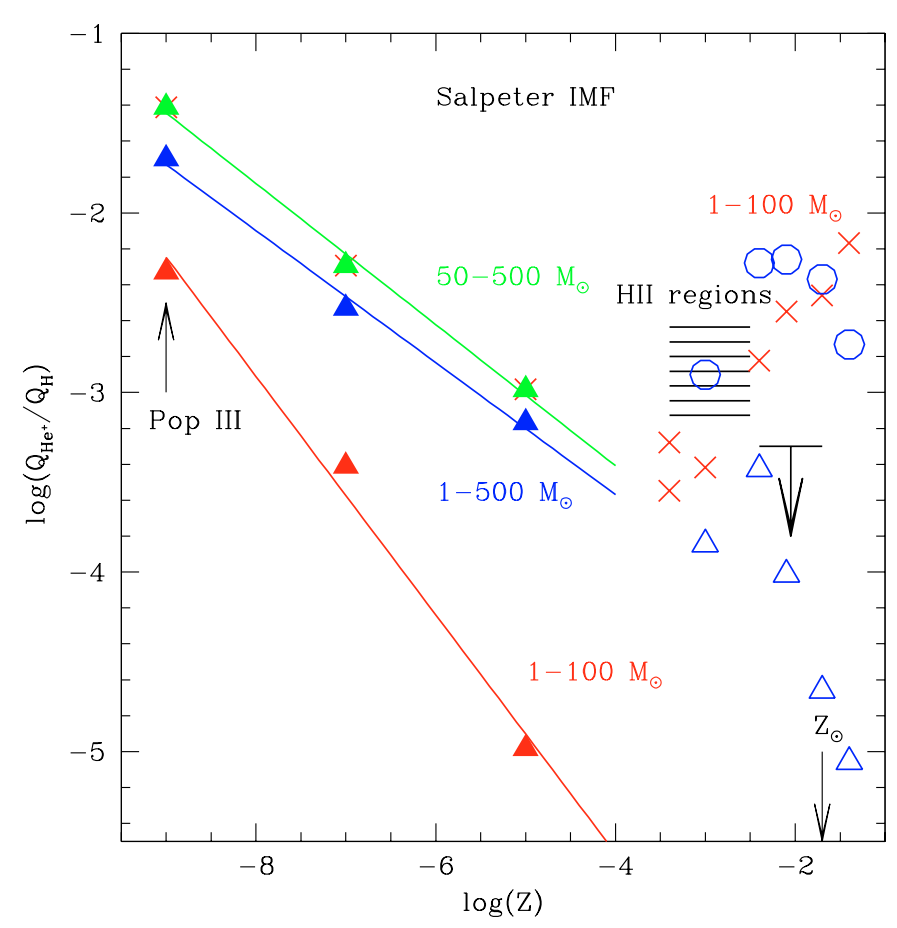

Fig. 5. Hardness $Q\left(\mathrm{He}^{+}\right) / Q(\mathrm{H})$ of the $\mathrm{He}^{+}$ionising flux for constant star formation as a function of metallicity (in mass fraction) for all models given in Table 4 . At metallicities above $Z \geq 4 \times 10^{-4}$ the predictions from our models (crosses), as well as those of Leitherer et al. (1999, open circles), and Smith et al. (2002, open triangles) are plotted. The shaded area and the upper limit (at higher $Z$ ) indicates the range of the empirical hardness estimated from $\mathrm{H}$ II region observations (see Sect. 6.3). At very low $Z$ solid lines show the fits to the data given by Eq. (2). Discussion in text.

$Q\left(\mathrm{He}^{+}\right) / Q(\mathrm{H})$ - before and during the WR phase. To circumvent this theoretical uncertainty we will subsequently derive an empirical estimate of the hardness at metallicities $Z \gtrsim 4 \times 10^{-4}$ (Sect. 6.3).

\subsubsection{Constant star formation}

For the case of constant star formation at equilibrium the metallicity dependence of the hardness $Q\left(\mathrm{He}^{+}\right) / Q(\mathrm{H})$ of the ionising flux is shown in Fig. 5 for all the IMFs considered. As apparent, for the very metal-poor cases $\left(Z \lesssim 10^{-4}\right) Q\left(\mathrm{He}^{+}\right) / Q(\mathrm{H})$ can be well fitted by

$\log \left(Q\left(\mathrm{He}^{+}\right) / Q(\mathrm{H})\right)=a \times \log (Z)+b$

with the numerical coefficients listed in Table 5, again taking into account that all metallicities $Z \leq Z_{\mathrm{lim}} \approx 10^{-9}$ are equivalent.

At higher metallicities - in the $Z$ range of known objects the theoretical predictions for $Q\left(\mathrm{He}^{+}\right) / Q(\mathrm{H})$ are probably less clear, due to possible presence of hot WR stars, difficulties in their modeling (cf. above), and the neglect of non-stellar emission processes which could contribute especially to $Q\left(\mathrm{He}^{+}\right)$. Indeed as shown in Fig. 5 rather important differences are obtained between various evolutionary synthesis models (SV98, Starburst 99 of Leitherer et al. 1999, and the latest computations of Smith et al. 2002). In the metallicity range $Z \sim 8 \times 10^{-4}$ to $5 \times 10^{-3}$ our empirical estimate of $Q\left(\mathrm{He}^{+}\right) / Q(\mathrm{H})$ (Sect. 6.3), 


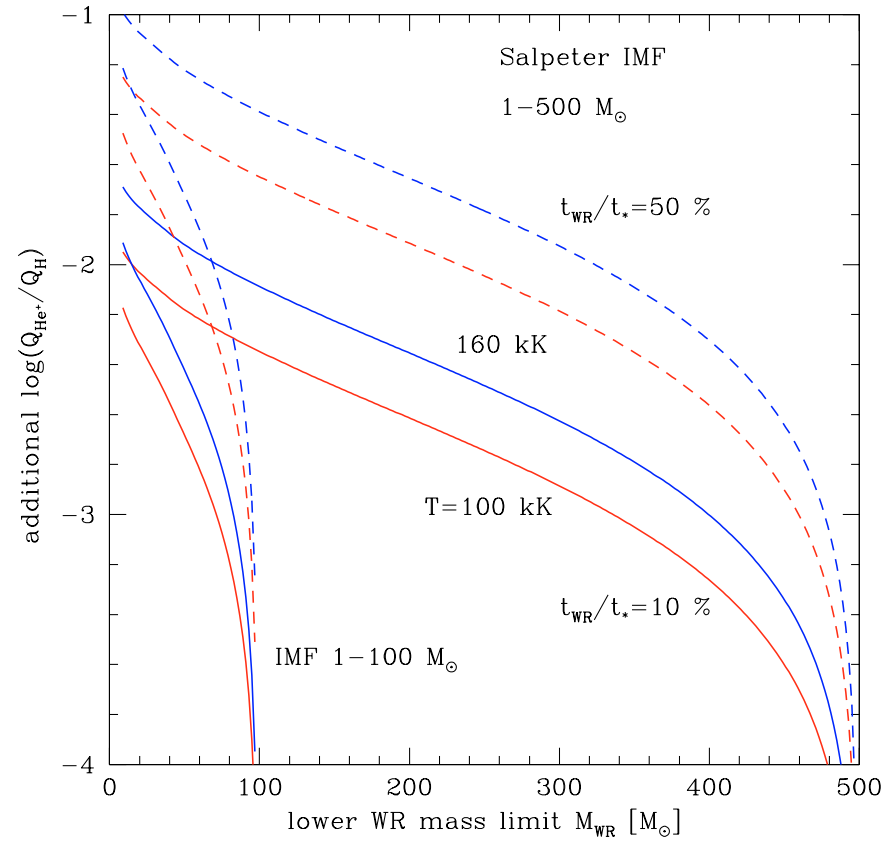

Fig. 6. Additional contribution $Q\left(\mathrm{He}^{+}\right)^{\star} / Q(\mathrm{H})$ of putative hot WRlike stars to the total $\mathrm{He}^{+}$hardness of the ionising flux $Q\left(\mathrm{He}^{+}\right) / Q(\mathrm{H})$ (cf. Eq. (6)) for SFR = const. as a function of the minimum mass $M_{\mathrm{WR}}$. Shown are the cases for $t_{\mathrm{WR}} / t_{\star}=10$ and $50 \%$ (solid and dashed curves), temperatures $T=100$ and $160 \mathrm{kK}$ (lower and upper curve respectively), and $M_{\text {up }}=500(100) M_{\odot}$ extending over the full plot (lower left only).

also shown in this figure, is likely more reliable than the models. At still larger $Z$ the average ionising spectrum of starbursts should be softer, as indicated by the tentative empirical upper limit and predicted by the Smith et al. (2002) models.

\subsection{Uncertainties on the $\mathrm{He}^{+}$ionising flux at very low metallicities $\left(Z \lesssim 10^{-4}\right)$}

In contrast to the Lyman continuum flux $Q(\mathrm{H})$, the $\mathrm{He}^{+}$ionising flux (and in general spectral features at high energy) show a very strong dependence on the stellar temperature in the $T_{\text {eff }}$ range $\sim 50-100 \mathrm{kK}$ typical of very low metallicity stars (Fig. 2 in S02). Therefore their prediction is naturally more sensitive to even small modifications of the exact stellar $T_{\text {eff }}$ or evolutionary scenario.

For example, one may wonder how reliable the above predictions of the metallicity dependence of $Q\left(\mathrm{He}^{+}\right) / Q(\mathrm{H})$ (Fig. 5) are at $Z \lesssim 10^{-4}$, where presently no observational constraints are available. In fact, studies of massive stars in the Local Group suggest that their average rotation rates increase towards low Z (Maeder et al. 1999), which - when combined with their increased compactness - can lead to non-negligible mass loss despite the low metallicity (Maeder \& Meynet 2000; Meynet \& Maeder 2002). If this effect is large enough, one could imagine that fast rotators could loose sufficient mass to follow a WR star like evolution leading possibly to a $\mathrm{He} / \mathrm{C} / \mathrm{O}$ core at temperatures $T \gtrsim 100 \mathrm{kK}$, a scenario known for metal-rich massive WR (e.g. Maeder \& Meynet 1988). Despite high rotational velocities, the detailed calculations of Meynet \& Maeder (2002) for $Z=10^{-5}$ do not show important alterations of the evolution for stars with $M \leq 60 M_{\odot}$. Exploratory calculations of Marigo et al. (2002) for Pop III stars treating in an simplified manner the effects of rotation on stellar mass loss find such a scenario for stars with initial mass $\gtrsim 750 M_{\odot}$.

Quantitatively the effect of such a putative hot "WR-like" population on the hardness of the ionising flux can be estimated for the case of constant star formation only in the following way. Suppose that stars of given initial mass $M \geq M_{\mathrm{WR}}$ spend this phase at constant luminosity $L$ and (hot) temperature $T$ during a constant fraction $f_{\mathrm{WR}}=\left(t_{\mathrm{WR}} / t_{\star}\right)$ of their lifetime $t_{\star}$. Assuming their winds are optically thin at $\geq 54 \mathrm{eV}$, the $\mathrm{He}^{+}$flux in this phase is then

$Q\left(\mathrm{He}^{+}\right)^{\star}(M)=\frac{L(M)}{\sigma T^{4}} q\left(\mathrm{He}^{+}\right)^{\star}(T)$,

where $q\left(\mathrm{He}^{+}\right)^{\star}$ is the photon flux per unit surface area (cf. Fig. 2 in $\mathrm{S} 02)$. The hardness $\left(Q\left(\mathrm{He}^{+}\right) / Q(\mathrm{H})\right)^{\prime}$ of a population including both the "normal" (main sequence) population and the additional hot population can be approximated by (for $\mathrm{SFR}=$ const.)

$\left(\frac{Q\left(\mathrm{He}^{+}\right)}{Q(\mathrm{H})}\right)^{\prime} \approx \frac{Q\left(\mathrm{He}^{+}\right)}{Q(\mathrm{H})}\left(1-f_{\mathrm{WR}}\right)+\frac{Q\left(\mathrm{He}^{+}\right)^{\star}}{Q(\mathrm{H})} f_{\mathrm{WR}}$,

assuming small changes in the total Lyman continuum photon output $Q(\mathrm{H})$. Here the first term stands for the unperturbed "normal" hardness shown in Fig. 5. The second term describes the additional contribution due to hot WR-like objects, which can be expressed as

$\frac{Q\left(\mathrm{He}^{+}\right)^{\star}}{Q(\mathrm{H})}=\frac{\int_{M_{\mathrm{wR}}}^{M_{\mathrm{up}}} t_{\star}(M) Q\left(\mathrm{He}^{+}\right)^{\star}(M) \Phi(M) \mathrm{d} M}{\int_{M_{\mathrm{low}}}^{M_{\mathrm{up}}} t_{\star}(M) \bar{Q}(\mathrm{H})(M) \Phi(M) \mathrm{d} M}$

where $Q$ have $(M)$ is the lifetime average of the Lyman continuum production, and $\Phi(M)$ is the IMF. The quantities $t_{\star}(M)$ and $\bar{Q}(\mathrm{H})(M)$ are taken from $\mathrm{S} 02$, and we assume as first approximation a luminosity $L(M)$ corresponding to the ZAMS ${ }^{4}$. The hardness contribution $Q\left(\mathrm{He}^{+}\right)^{\star} / Q(\mathrm{H})$ is then computed assuming temperatures $T=100-160 \mathrm{kK}$ as expected from stellar evolution models, and durations $f_{\mathrm{WR}}=\left(t_{\mathrm{WR}} / t_{\star}\right)$ of 0.1 or 0.5 . The result is plotted for the IMFs A and B $\left(M_{\text {up }}=100\right.$ and $500 M_{\odot}$ ) in Fig. 6 as a function of the minimum mass $M_{\mathrm{WR}}$, above which all stars are assumed to reach this hot "WR" phase.

The case of $\left(t_{\mathrm{WR}} / t_{\star}\right)=50 \%$, which would require very strong mass loss already during the main sequence or a nearly homogeneous evolution leading early to a blueward evolution, appears extremely unlikely and is shown here to mimic the "strong mass loss" models adopted in the Pop III models of S02. For a hot phase of a duration typical of the post main sequence evolution ( $\sim 10 \%$ of total lifetime) Fig. 6 shows that such putative "hot WR" could in the "best" case contribute an additional hardness $Q\left(\mathrm{He}^{+}\right)^{\star} / Q(\mathrm{H})$ of the order of $10^{-2 \ldots-3}$, comparable to the hardness of normal stellar populations with metallicities $Z \lesssim 10^{-4}$ (cf. Fig. 5). To examine how realistic

${ }^{4}$ The results discussed below are fairly insensitive to the exact (low) metallicity adopted for these quantities. 
Table 5. Fit coefficients for Eq. (2).

\begin{tabular}{lll}
\hline \hline IMF & $a$ & $b$ \\
\hline A & $-0.66 \pm 0.071$ & $-8.22 \pm 0.51$ \\
B & $-0.37 \pm 0.028$ & $-5.04 \pm 0.20$ \\
C & $-0.39 \pm 0.028$ & $-4.98 \pm 0.20$ \\
\hline
\end{tabular}

such cases may be, will require a detailed understanding of the coupled processes of stellar mass loss, rotation, and internal mixing. At present the available limits are $M_{\mathrm{WR}}>60 M_{\odot}$ at $Z=10^{-5}$ and $\left(t_{\mathrm{WR}} / t_{\star}\right) \lesssim 10 \%$ from the rotating stellar models of Meynet \& Maeder (2002), and $M_{\mathrm{WR}} \gtrsim 750 M_{\odot}$ at $Z=0$ from the simplified models of Marigo et al. (2002).

Although the above exercise shows that at very low metallicity $\left(Z \lesssim 10^{-5}\right)$ the hardness $Q\left(\mathrm{He}^{+}\right) / Q(\mathrm{H})$ due to stellar sources could be higher than shown in Fig. 5, it seems that such scenarios are quite unlikely. If star formation takes place on much longer time scales, and massive stars would not form (or in much smaller quantities), hot planetary nebulae could also be a source of hard ionising photons, as illustrated by the scenario of Shioya et al. (2002). In any case, a major uncertainty stems from our limited knowledge of the IMF at very low metallicities.

\subsection{Empirical constraints on the $\mathrm{He}^{+}$ionising flux of starbursts at $Z \gtrsim 4 \times 10^{-4}$}

Spectroscopic observations of extra-galactic giant $\mathrm{H}$ II regions probing He II recombinations lines can yield empirical information on the "average" hardness $Q\left(\mathrm{He}^{+}\right) / Q(\mathrm{H})$ of starbursts. Indeed it is well known that a fairly large fraction of metal-poor $\mathrm{H}$ II regions show the presence of nebular He II $\lambda 4686$ emission indicative of a hard ionising spectrum (see e.g. Guseva et al. 2000; compilation of Schaerer et al. 1999). A complete explanation of the origin of the required high energy photons (shocks, X-rays, WR stars) remains to be found (e.g. Garnett et al. 1991; Schaerer 1996, 1998; Guseva et al. 2000; Izotov et al. 2001; Stasińska \& Izotov 2002).

The largest sample of high quality data is that of Izotov and collaborators (cf. Guseva et al. 2000 and references therein), which shows He II $\lambda 4686$ detections with typical relative intensities of $I(4686) / I(\mathrm{H} \beta) \sim 1-2 \%$.

From such a sample we may estimate an average hardness from

$$
\frac{Q\left(\mathrm{He}^{+}\right)}{Q(\mathrm{H})}=\frac{I(4686)}{I(\mathrm{H} \beta)} \times a \times f_{\text {detect }} \times \frac{t_{\text {detect }}}{t_{\text {total }}},
$$

where $a\left(=0.47(0.63)\right.$ for $\left.T_{\mathrm{e}}=10000(30000) \mathrm{K}\right)$ translates the relative line emissivities, $f_{\text {detect }}$ is the fraction of objects showing nebular He II among the spectra of sufficient quality to allow detections down to this intensity level, $t_{\text {detect }}$ is the age range covered by the observed $\mathrm{H}$ II regions, and $t_{\text {total }}$ is the total lifetime of $\mathrm{H}$ II regions. Inspection of the data of Izotov yields $f_{\text {detect }} \sim 1 / 3-1 / 2$ for metallicities $Z \in\left[8 \times 10^{-4}, 4.8 \times 10^{-3}\right]$ and possibly even a larger fraction at the lowest observed metallicities. (Stasińska \& Izotov 2002). One has $t_{\text {total }} \sim 10 \mathrm{Myr}$, the typical $\mathrm{H}$ in region lifetime or the time over which bursts show detectable line emission. However, it is now well established that $\mathrm{H}$ II region samples suffer from selection biases leading to an absence of advanced bursts, or in other words a preferential selection of bursts with ages younger than $\lesssim 4$ 5 Myr (e.g. Bresolin et al. 1999; Raimann et al. 2000; Moy et al. 2001; Stasińska et al. 2001). For this range of values and $a=0.47$ one obtains an estimate of $\log \left(Q\left(\mathrm{He}^{+}\right) / Q(\mathrm{H})\right) \sim-3.2$ to -2.6. Postulating the extreme case that additionally the first $2 \mathrm{Myr}$ of the $\mathrm{H}$ II phase are also not detected in the optical ${ }^{5}$ one has a minimum value of $t_{\text {detect }} \sim 1 \mathrm{Myr}$, yielding a lower limit of $\log \left(Q\left(\mathrm{He}^{+}\right) / Q(\mathrm{H})\right) \gtrsim-3.5$. In principle this average value could be even larger as the (conservative) assumption of $t_{\text {detect }} / t_{\text {total }}<1$ implies that older (non-detected) $\mathrm{H}$ II regions have no $\mathrm{He}^{+}$ionising flux.

This estimate is obviously independent of the nature of the hard $\left(\mathrm{He}^{+}\right)$ionising radiation. By construction Eq. (6) provides an estimate of the average $Q\left(\mathrm{He}^{+}\right) / Q(\mathrm{H})$ expected in objects with constant ongoing star formation for metallicities $Z \sim 8 \times$ $10^{-4}$ to $5 \times 10^{-3}$. The near absence of nebular He II detections in $\mathrm{H}$ II regions at higher metallicity (cf. Schaerer 1998; Guseva et al. 2000) indicates softer spectra. However, it is difficult to establish a firm upper limit on $Q\left(\mathrm{He}^{+}\right) / Q(\mathrm{H})$ for $Z \gtrsim 5 \times 10^{-3}$. We here retain $\log \left(Q\left(\mathrm{He}^{+}\right) / Q(\mathrm{H})\right) \ll-3.3$ as a tentative limit.

\subsection{Overall behaviour of $Q\left(H e^{+}\right) / Q(H)$ with metallicity}

In short, from the considerations above, we find the following two cases for the most plausible metallicity dependence of the average $Q\left(\mathrm{He}^{+}\right) / Q(\mathrm{H})$ hardness ratio of starbursts with metallicity (see Fig. 5).

1) If a universal Salpeter like IMF with a "normal" upper mass limit of $\sim 100 M_{\odot}$ prevails for all metallicities the hardness decreases by more than 2 orders of magnitude from $Z=0$ to $\sim 10^{-4}$, re-increases thereafter (up to $Z \lesssim 5 \times 10^{-3}$, in metalpoor starbursts) to a level $\sim 2$ to 10 times smaller than that of Pop III objects, and decreases again to low levels for higher metallicities.

2) If very massive stars are favoured at metallicities $Z \lesssim$ $10^{-4}$, the hardness $Q\left(\mathrm{He}^{+}\right) / Q(\mathrm{H})$ of Pop III objects is considerably enhanced (corresponding to a powerlaw spectrum with spectral index $\alpha \sim 2.3-2.8$ in $F_{v}$ ), then decreases down to levels somewhat smaller or comparable to that of metal-poor starbursts, before decreasing further to levels at least two orders of magnitude softer than at zero metallicity.

\section{Ly $\alpha$ and $\mathrm{He}$ II $\lambda 1640$ emission at various metallicities}

To first order recombination line luminosities are proportional to the ionising photon flux and are thus simply expressed as (in units of $\mathrm{erg} \mathrm{s}^{-1}$ )

$L_{\mathrm{l}}(t)\left[\mathrm{erg} \mathrm{s}^{-1}\right]=c_{1}\left(1-f_{\mathrm{esc}}\right) Q_{\mathrm{i}}(t)\left[\mathrm{s}^{-1}\right]$

where $c_{1}$ are the line emission coefficients given in Table 1, $f_{\text {esc }}$ is the photon escape fraction out of the galaxy or observed

\footnotetext{
5 The well known absence of $\mathrm{H}$ II regions with very large $\mathrm{H} \beta$ equivalent widths could be taken as an indications for such a scenario.
} 

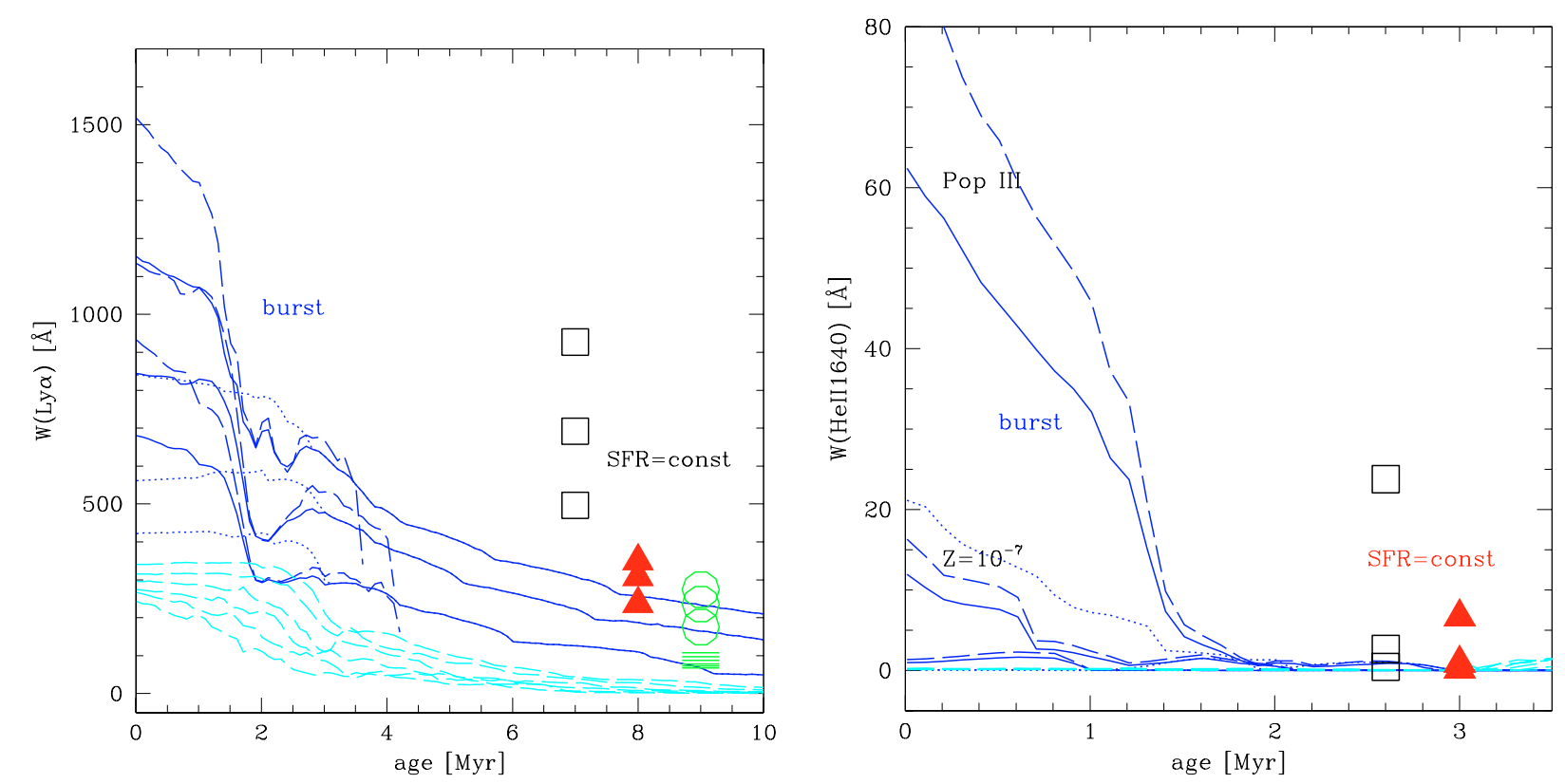

Fig. 7. Temporal evolution of the Ly $\alpha$ equivalent width (left panel) and He II $\lambda 1640$ equivalent width (right) for instantaneous bursts at all metallicities. The very metal-poor models $\left(Z=0,10^{-7}\right.$, and $\left.10^{-5}\right)$ with the IMFs C (50-500 $\left.M_{\odot}\right)$, B $\left(1-500 M_{\odot}\right)$ and A $\left(1-100 M_{\odot}\right)$ are shown as short-dashed, solid, and dotted lines respectively from top to bottom. The remaining metallicities (for IMF A) are shown with dashed lines. The equilibrium values for SFR = const. at metallicities $Z \leq 10^{-5}$ are plotted on the right (at arbitrary ages) using open squares for the IMF C, filled triangles for IMF B, open circles for IMF A, and using short lines for higher metallicities (with IMF A). Note the very large maximum $W(\operatorname{Ly} \alpha)$ predicted at young ages. $W(\mathrm{He}$ II $\lambda 1640) \gtrsim 5 \AA$ are only expected at the lowest metallicities $\left(Z \lesssim 10^{-7}\right)$, except if hot WR-like stars not included in the tracks were formed e.g. through important stellar mass loss (Sect. 6.2). See text for further discussion.

aperture, and $Q_{\mathrm{i}}$ is the ionising photon flux (in units of $\mathrm{s}^{-1}$ ) corresponding to the appropriate recombination line.

However, as is well known, Ly $\alpha$ constitutes a particular case due to its very large line optical depth, which implies that several effects (e.g. dust absorption, ISM geometry and velocity structure) can alter the total Ly $\alpha$ emission and lead to complex line profiles (cf. Charlot \& Fall 1993; Valls-Gabaud 1993; Chen \& Neufeld 1994; Kunth et al. 1998; Loeb \& Rybicki 1999; Tenorio-Tagle et al. 1999). Furthermore for Ly $\alpha$ source at redshifts close to or above the redshift of reionisation the intrinsic Ly $\alpha$ emission may be further reduced or suppressed by absorption in nearby line of sight HI clouds (cf. MiraldaEscude \& Rees 1997; but also Haiman 2002; Madau 2002). A proper treatment of these effects requires a complex solution of radiation transfer which depends strongly on geometrical properties of the ISM and IGM, and for which no general solution is possible. One must thus caution that depending on the application our simplifying assumptions may not apply and the predicted Ly $\alpha$ emission should thus be considered as an upper limit. Note that these difficulties do not affect other recombination lines such as $\mathrm{H} \alpha$ and $\mathrm{He}$ II $\lambda 1640$, whose optical depth is strongly reduced compared to $\operatorname{Ly} \alpha$.

Bearing the above in mind, the time evolution of the $\operatorname{Ly} \alpha$ and $\mathrm{He}$ II $\lambda 1640$ line luminosities can be deduced from the evolution of $Q(\mathrm{H})$ and $Q\left(\mathrm{He}^{+}\right)$respectively given in Fig. 3 .

In the case of constant star formation, at equilibrium, recombination line luminosities $L_{1}$ are proportional to the star formation rate (SFR), i.e.

$L_{1}=\left(1-f_{\mathrm{esc}}\right) f_{1}\left(\frac{\mathrm{SFR}}{M_{\odot} \mathrm{yr}^{-1}}\right)$.
For $L_{1}$ in erg $\mathrm{s}^{-1}$ the proportionality constants $f_{1}$ for $\mathrm{Ly} \alpha, \mathrm{H} \alpha$, and He II $\lambda 1640$ are listed in Cols. 9-11 of Table 4. The variations of the ionising flux $Q(\mathrm{H})$ already discussed above imply in particular lower star formation rates at low metallicity when identical recombination lines are used. We note that our $\mathrm{H} \alpha$ SFR conversion factors are in good agreement with other computations at various metallicities (e.g. Kennicutt 1998; Sullivan et al. 2001) when rescaled for their $M_{\text {low }}$ using $c_{\mathrm{M}}$ (Table 2).

The predicted Ly $\alpha$ and $\mathrm{He}$ II $\lambda 1640$ emission line equivalent widths of ageing bursts of different metallicities and all the IMF cases considered are shown in Fig. 7. Note that our revised Pop III models show smaller Ly $\alpha$ equivalent widths compared to the previous calculation in S02. This is due to an erroneous continuum definition in the earlier computations. The new models supercede those of S02. Good agreement is also found with the $W(\operatorname{Ly} \alpha)$ predictions of Tumlinson et al. (2002). The reader is also reminded that none of these recent calculations include stellar Ly $\alpha$ absorption (cf. Valls-Gabaud 1993; Charlot \& Fall 1993; and Valls-Gabaud \& Schaerer 2002 for new predictions).

Maximum Ly $\alpha$ equivalent widths of $\sim 240-350 \AA$ are predicted for metallicities between solar and $4 \times 10^{-4}$. For $Z$ down to zero (Pop III), $\max (W(\operatorname{Ly} \alpha))$ may reach up to $\sim 800$ $1500 \AA$ for the various adopted IMFs (cf. S02) For comparison, the equilibrium values for SFR = const. are in the range of $W(\operatorname{Ly} \alpha) \sim 175-275,240-350,500-930 \AA$ for IMF A, B, and C respectively at $Z \leq 10^{-5}$, and $\sim 70-100 \AA$ for higher metallicities (IMF A). Note that the increased Lyman continuum output of young very metal-poor populations alone does not explain the strong increase of $W(\operatorname{Ly} \alpha)$ (cf. Table 3). In addition 
the reduced stellar UV continuous luminosity at $\lambda \sim 1215 \AA$, due to the shift of the SED peak far into the Lyman continuum (Fig. 2), also contributes to increase $W(\operatorname{Ly} \alpha)$.

A high median Ly $\alpha$ equivalent width $(\sim 430 \AA)$ was found in the Large Area Lyman Alpha (LALA) survey of Malhotra \& Rhoads (2002) at $z=4.5$ and interpreted as due to AGN, starbursts with flat IMFs, or even Pop III objects. Indeed, if constant star formation is appropriate for their objects and the IMF slope is universally that of Salpeter, the observed large $W(\operatorname{Ly} \alpha)$ would require very metal-poor populations with large upper mass cut-offs and/or an increased lower cut-off (e.g. IMFs B or C). Alternatively their observations could also be explained by predominantly young bursts, with metallicities $\lesssim 10^{-5}$ and no need for extreme IMFs (Fig. 7). This issue will be addressed in detail in a subsequent publication (Valls-Gabaud \& Schaerer 2002).

As expected from the softening of the radiation field with increasing metallicity, the $\mathrm{He}$ II $\lambda 1640$ equivalent widths decreases strongly with $Z$; values $W(\mathrm{He}$ II $\lambda 1640) \gtrsim 5 \AA$ are expected only at metallicities $Z \lesssim 10^{-7}$, except if hot WR-like stars (cf. Sect. 6.2) or non-stellar sources (e.g. X-rays, AGN) provide sufficient amounts of $\mathrm{He}^{+}$ionising photons.

Part of the relative weakness of $W(\mathrm{He}$ II $\lambda 1640)$ compared to $W(\operatorname{Ly} \alpha)$ is due to a relatively strong, mostly nebular, continuum flux at $1640 \AA$ (see S02). As Ly $\alpha$ emission may be strongly reduced due to the effects discussed earlier in objects beyond the re-ionisation redshift, and the $\mathrm{He}$ II $\lambda 1640$ luminosity is potentially strong enough to be detected (cf. Tumlinson et al. 2001; Oh et al. 2001; Schaerer \& Pelló 2001), it is a priori not clear if both lines may be observed simultaneously and if so which of the two lines would be stronger.

\section{Conclusions}

We have examined the spectral properties of the ionising continua, the Lyman-break, and the Ly $\alpha$ and He II $\lambda 1640$ recombination lines in starbursts with metallicities $Z$ from zero - corresponding to primordial, Pop III objects - over low metallicities $\left(Z \gtrsim 4 \times 10^{-4}\right)$ observed in nearby galaxies, up to solar metallicity $\left(Z_{\odot}=0.02\right)$.

Our computations, including new sets of stellar evolution models at very low metallicities $\left(Z=10^{-7}, 10^{-5}\right)$ and previously published grids at other $Z$, allow us in particular to study how spectral properties vary in the transition from Pop III objects to "normal" currently measurable metallicities.

Various IMFs are treated, including also cases where very massive stars (up to $\sim 500 M_{\odot}$ ) are formed, as suggested by hydrodynamical calculations for metallicities $Z \lesssim Z_{\text {crit }} \approx 10^{-5}$ (Bromm et al. 2001a; Abel et al. 1998; Nakamura \& Umemura 2001).

Predictions are provided for the number of $\mathrm{H}, \mathrm{He}^{0}$, and $\mathrm{He}^{+}$ionising photons and average photon energies in these continua, the hardness of the ionising spectrum, the amplitude of the Lyman-break, the number of photons able to photodissociate $\mathrm{H}_{2}$, and finally recombination line luminosities and equivalent widths (mostly for Ly $\alpha$ and $\mathrm{He}$ II $\lambda 1640$ ).

Two limiting cases of star formation histories, instantaneous bursts and constant star formation, are considered. For
SFR $=$ const., presumably appropriate to describe the average properties of starbursts galaxies or populations thereof, the following main results have been obtained:

- As expected from numerous earlier computations the Lyman continuum flux $Q(\mathrm{H})$ increases with decreasing metallicity. For a universal Salpeter IMF from 1-100 $M_{\odot}$ the enhancement reaches typically a factor of $\sim 3$ between solar metallicity and Pop III objects for a constant star formation (Sect. 4, cf. Tumlinson \& Shull 2000; Schaerer 2002).

- While for metallicities $Z \gtrsim 1 / 50 Z_{\odot}$ the amplitude of the Lyman-break is rather metallicity independent, a reduction by a factor $\sim 2$ is found at lower metallicities, related to the strong increase of the average stellar temperature (Sect. 5).

- The predicted hardness of the ionising fluxes between $\geq 54 \mathrm{eV}$ and $\geq 13.6 \mathrm{eV}$, i.e. the ability to doubly ionise $\mathrm{He}$, decreases by $\sim 1.5-2$ or more orders of magnitude from $Z=0$ to $\sim 10^{-4}$ depending strongly on the upper mass cutoff of the IMF (Sect. 6).

From empirical constraints we derive a hardness $\log Q\left(\mathrm{He}^{+}\right) / Q(\mathrm{H}) \sim-3.2$ to -2.6 for metal-poor starbursts $\left(1 / 25 \lesssim Z / Z_{\odot} \lesssim 1 / 4\right)$ and softer spectra for higher metallicities (Sect. 6.3). The former should provide the best estimate of $Q\left(\mathrm{He}^{+}\right) / Q(\mathrm{H})$; the latter finding is also compatible with recent evolutionary synthesis models of Smith et al. (2002) including line blanketed non-LTE atmospheres for WR and O stars.

We also provide (Sect. 6.2) a simple estimate of the possible impact of hot WR like stars on $Q\left(\mathrm{He}^{+}\right) / Q(\mathrm{H})$ at very low metallicities $\left(Z \lesssim 10^{-4}\right)$. Such stars could eventually form due to a strong enhancement of mass loss related to rapid rotation (Marigo et al. 2002) or in principle also due very efficient rotational mixing processes (cf. Meynet \& Maeder 2002), although we believe that these scenarios are quite unlikely or overall are of minor importance.

- Finally, calibrations for star formation rate determinations from Ly $\alpha$ and other recombination lines at all metallicities and for various IMFs are provided (Sect. 7).

For young bursts, the maximum $\operatorname{Ly} \alpha$ equivalent width predicted is shown to increase strongly with decreasing metallicity from $W(\operatorname{Ly} \alpha) \sim 240-350 \AA$ at $Z \gtrsim 1 / 50 Z_{\odot}$ to $400-850 \AA$ or higher at $Z$ between $10^{-5}$ and 0 (Pop III) for the same Salpeter IMF (Sect. 7).

We find that non-negligible $\mathrm{He}$ II $\lambda 1640$ emission due to photoionisation from stellar sources appears to be limited to very small metallicities $\left(\log \left(Z / Z_{\odot}\right) \lesssim-5.3\right)$ and Population III objects, except if hot WR like stars, whose existence appears very speculative, were frequent.

The detailed model predictions presented here are available on the Web through the CDS and at http://webast.ast.obs-mip.fr/sfr/. In subsequent publications our models are applied to the interpretation of Ly $\alpha$ observations in high redshift galaxies (Valls-Gabaud \& Schaerer 2002), modeling of the combined chemical enrichment and re-ionisation history of the Universe (Ferrara \& Schaerer 2002), and feasibility studies on the detection of Population III objects (Pelló \& Schaerer 2002). 
Acknowledgements. I thank Andrea Ferrara, Roser Pelló, David VallsGabaud for stimulating discussions and comments on an earlier version of the manuscript. Useful comments from Tom Abel, Daniel Kunth, and Grazyna Stasińska on the draft and related issues were also appreciated. Richard Norris kindly provided model results from his calculations for comparison. Last, but not least, I thank the referee for useful comments which helped to improve the presentation.

\section{References}

Abel, T., Anninos, P. A., Norman, M. L., \& Zhang, Y. 1998, ApJ, 508, 518

Abel, T., Bryan, G. L., \& Norman, M. L. 2002, Science, 295, 93

Ajiki, M., et al. 2002, ApJ, in press [astro-ph/0207303]

Bresolin, F., Kennicutt, R. C., \& Garnett, D. R. 1999, ApJ, 510, 104

Bromm, V., Coppi, P. S., \& Larson, R. B. 1999, ApJ, 527, L5

Bromm, V., Ferrara, A., Coppi, P. S., \& Larson, R. B. 2001a, MNRAS, 328, 969

Bromm, V., Kudritzki, R. P., \& Loeb, A. 2001b, ApJ, 552, 464

Charlot, S., \& Fall, M. S. 1993, ApJ, 415, 580

Chen, W. L., \& Neufeld, N. A. 1994, ApJ, 432, 567

Ciardi, B., Ferrara, A., Governato, F., \& Jenkins, A. 2000, MNRAS, 314,611

Deharveng, J.-M., Faiesse, S., Le Brun, V., \& Milliard, B. 1997, A\&A, 325,1259

Deharveng, J.-M., Buat, V., Le Brun, V., et al. 2001, A\&A, 375, 805

Desjacques, V. 2000, Diploma Thesis, Geneva Observatory

El Eid, M. F., Fricke, K. J., \& Ober, W. W. 1983, A\&A, 119, 54

Ellis, R., Santos, M. R., Kneib, J.-P., \& Kuijken, K. 2001, ApJ, 560, L119

Feijóo, J. M. 1999, Diploma thesis, Geneva Observatory

Frye, B., Broadhurst, T., \& Benítez, N. 2002, ApJ, 568, 558

Ferrara, A., \& Schaerer, D. 2002, in preparation

Garnett, D. R., Kennicutt, R. C. J., Chu, Y.-H., \& Skillman, E. D. 1991, ApJ, 373, 458

Gnedin, N. Y. 1998, ApJ, MNRAS, 294, 407

Gnedin, N. Y., \& Ostriker, J. P. 1997, ApJ, 486, 581

Guseva, N. G., Izotov, Y. I., \& Thuan, T. X. 2000, ApJ, 531, 776

Haiman, Z. 2002, ApJ, 576, L1

Hernandez, X., \& Ferrara, A. 2001, MNRAS, 324, 484

Hu, E. M., Cowie, L. L., McMahon, R. G., et al. 2002, ApJ, 568, L75

Hu, E. M., Cowie, L. L., \& McMahon, R. G. 1998, ApJ, 502, L99

Hu, E. M., McMahon, R. G., \& Cowie, L. L. 1999, ApJ, 522, L9

Hubeny, I., \& Lanz, T. 1995, ApJ, 439, 875

Izotov, Y. I., Chaffe, F. H., \& Schaerer, D. 2001, A\&A, 378, L45

Kennicutt, R. C. 1998, ARA\&A, 36, 189

Kudritzki, R. P. 2002, ApJ, 577, 389

Kudritzki, R.-P., Méndez, R. H., Feldmeier, J. J., et al. 2000, ApJ, 536, 19

Kunth, D., Mas-Hesse, J. M., et al. 1998, A\&A, 334, 11

Kurucz, R. L. 1991, in Stellar Atmospheres: Beyond Classical Models, NATO ASI Ser. C, vol. 341, ed. L. Crivellari, I. Hubeny, \& D. G. Hummer, 441

Leitherer, C. 1998, in The Stellar Initial Mass Function, ASP Conf. Ser., 142, 61

Leitherer, C., Ferguson, H. C., Heckman, T. M., \& Lowenthal, J. 1995, ApJ, 454, L19
Leitherer, C., Schaerer, D., Goldader, J. D., et al. 1999, ApJS, 123, 3

Lejeune, T., \& Schaerer, D., A\&A, 366, 538

Loeb, A., \& Barkana, R. 2001, ARA\&A, 39, 19

Loeb, A., \& Rybicki, G. B. 1999, ApJ, 524, 527

Madau, P. 2002, IAU Symp. 212, ed. K. van der Hucht, A. Herrero, \& C. Esteban, in press

Maeder, A., Grebel, E. K., \& Mermilliod, J.-C. 1999, A\&A, 346, 459

Maeder, A., \& Meynet, G. 1994, A\&A, 287, 803

Maeder, A., \& Meynet, G. 2000, A\&A, 361, 159

Malhotra, S., \& Rhoads, J. E. 2002, ApJ, 565, L7

Marigo, P., Girardi, L., Chiosi, C., \& Wood, R. 2001, A\&A, 371, 152

Meynet, G., \& Maeder, A. 2002, A\&A, 390, 561

Mihalas, D. 1978, Stellar Atmospheres (W.H. Freeman \& Co., San Francisco)

Miralda-Escudé, J., \& Rees, M. J. 1997, ApJ, 478, L57

Moy, E., Rocca-Volmerange, B., \& Fioc, M. 2001, A\&A, 365, 347

Nakamura, F., \& Umemura, M. 2001, ApJ, 548, 19

Oh, S. P., Haiman, Z., \& Rees, M. J. 2001, ApJ, 553, 73

Pelló, R., \& Schaerer, D. 2002, in preparation

Raimann, D., Bica, E., Storchi-Bergmann, T., \& Melnick, J. 2000, MNRAS, 314, 295

Rhoads, J. E., \& Malhotra, S. 2001, ApJ, 563, L5

Schaerer, D. 1996, ApJ, 467, L17

Schaerer, D. 1998, Highlights in Astronomy, 11, 134

Schaerer, D. 2000, in Stars, Gas and Dust in Galaxies: Exploring the Links, ed. D. Alloin, K. Olsen, \& G. Galaz, ASP Conf. Ser., 221, 99

Schaerer, D. 2002a, A\&A, 382, 28 (S02)

Schaerer, D. 2002b, IAU Symp. 212, ed. K. van der Hucht, A. Herrero, $\&$ C. Esteban, in press [astro-ph/0208227]

Schaerer, D., Contini, T., \& Pindao, M. 1999, A\&AS, 136, 35

Schaerer, D., \& de Koter, A. 1997, A\&A, 322, 598

Schaerer, D., \& Vacca, W. D. 1998, ApJ, 497, 618 (SV98)

Schmutz, W., Leitherer, C., \& Gruenwald, R. 1992, PASP, 104, 1164

Shioya, Y., Taniguchi, Y., Murayama, T., et al. 2002, ApJ, 576, 36

Smith, L., Norris, R., \& Crowther, P. A. 2002, MNRAS, in press [astro-ph/0207554]

Spitzer, L. 1978, Physical Processes in the Interstellar Medium (Wiley, New York)

Stasińska, G., \& Izotov, Y. I. 2002, A\&A, submitted

Stasińska, G., \& Schaerer, D. 1999, A\&A, 351, 72

Stasińska, G., Schaerer, D., \& Leitherer, C. 2001, A\&A, 370, 1

Stasińska, G., \& Tylenda, R. 1986, A\&A, 155, 137

Steidel, C. C., Giavalisco, M., Dickinson, M., \& Adelberger, K. L. 1996, AJ, 112, 352

Steidel, C. C., Pettini, M., \& Adelberger, K. L. 2001, ApJ, 546, 665

Stern, D., \& Spinrad, H. 1999, PASP, 111, 1475

Storey, P. J., \& Hummer, D. G. 1995, MNRAS, 272, 41

Sullivan, M., Mobascher, B., Chan, B., et al. 2001, ApJ, 558, 72

Tenorio-Tagle, G., Silich, S. A., Kunth, D., Terlevich, E., \& Terlevich, R. 1999, MNRAS, 309, 332

Tumlinson, J., Giroux, M. L., \& Shull, J. M. 2001, ApJ, 550, L1

Tumlinson, J., \& Shull, J. M. 2000, ApJ, 528, L65

Tumlinson, J., Shull, J. M., \& Venkatesan, A. 2002, ApJ, submitted [astro-ph/0206389]

Valls-Gabaud, D. 1993, ApJ, 419, 7

Valls-Gabaud, D., \& Schaerer, D. 2002, in preparation 